\title{
Radiographic characteristics of the hand and cervical spine in fibrodysplasia ossificans progressiva
}

\author{
Kenichi Mishima ${ }^{1}$, Hiroshi Kitoh ${ }^{1,2, *}$, Nobuhiko Haga ${ }^{2}$, Yasuharu Nakashima ${ }^{2}$, Junji Kamizono ${ }^{2}$, \\ Takenobu Katagiri' ${ }^{2}$, Takafumi Susami ${ }^{2}$, Masaki Matsushita ${ }^{1}$, Naoki Ishiguro ${ }^{1}$ \\ ${ }^{1}$ Department of Orthopaedic Surgery, Nagoya University Graduate School of Medicine, Nagoya, Aichi, Japan; \\ ${ }^{2}$ The Research Committee on Fibrodysplasia Ossificans Progressiva, Tokyo, Japan.
}

\begin{abstract}
Summary Fibrodysplasia ossificans progressiva (FOP) is a disabling heritable disorder of connective tissue characterized by progressive heterotopic ossification in various extraskeletal sites. Early correct diagnosis of FOP is important to prevent additional iatrogenic harm or trauma. Congenital malformation of the great toes is a well-known diagnostic clue, but some patients show normal-appearing great toes. The thumb shortening and cervical spine abnormalities are other skeletal features often observed in FOP. This study aimed to address the quantitative assessment of these features in a cohort of patients with FOP, which potentially helps early diagnosis of FOP. Radiographs of the hand and cervical spine were retrospectively analyzed from a total of 18 FOP patients ( 9 males and 9 females) with an average age of 13.9 years (range $0.7-39.3$ years). The elevated ratio of the second metacarpal bone to the distal phalanx of the thumb $(>+1 \mathrm{SD})$ was a consistent finding irrespective of the patient's age and gender. Infant FOP patients, in addition, exhibited an extremely high ratio of the second metacarpal bone to the first metacarpal bone $(>+3 S D)$. The height/depth ratio of the $\mathrm{C} 5$ vertebra increased in patients over 4 years of age $(>+2 \mathrm{SD})$. Additionally, the ratio of (height+depth) of the $\mathrm{C} 5$ spinous process to the $\mathrm{C} 5$ vertebral depth was markedly elevated in young patients ( $>+2$ SD). We quantitatively demonstrated the hand and cervical spine characteristics of FOP. These findings, which can be seen from early infancy, could be useful for early diagnosis of FOP even in patients without great toe abnormalities.
\end{abstract}

Keywords: Fibrodysplasia ossificans progressiva, early diagnosis, radiographic characteristics

\section{Introduction}

Fibrodysplasia ossificans progressiva (FOP) is a severely disabling genetic disorder of connective tissues characterized by congenital malformations of the great toes and progressive heterotopic ossification $(\mathrm{HO})$ in various extraskeletal sites including muscles, tendons, ligaments, fascias, and aponeuroses. FOP is caused by a recurrent activating mutation (c.617G $>$ A, p.R206H) in the gene encoding activin receptor IA/activin-like kinase 2 (ACVR1/ALK2), a bone morphogenetic protein (BMP) type I receptor (1). HO typically begins

*Address correspondence to:

Dr. Hiroshi Kitoh, Department of Orthopaedic Surgery,

Nagoya University Graduate School of Medicine, 65

Tsurumai, Showa-ku, Nagoya, Aichi, 466-8550, Japan.

E-mail: hkitoh@med.nagoya-u.ac.jp to form during the first decade of life preceded by painful soft tissue swelling and inflammation (flareups), which are sometimes mistaken for aggressive fibromatosis or musculoskeletal tumors. Surgical resection of $\mathrm{HO}$ leads to explosive new bone formation (2). Since there is no definitive treatment to prevent progressive HO in FOP to date (3), early correct diagnosis is necessary to maintain their mobility by preventing additional iatrogenic harm (4).

Malformations of the great toes, such as hallux valgus, deformed proximal phalanges and shortened first metatarsal bones, are well-known pre-osseous features of FOP (5). A reported incidence of these deformities is $95 \%$, suggesting that there exists rare FOP cases without the great toe abnormalities (6). We demonstrated additional early radiographic signs of FOP including shortening of the first metacarpal bones and hypertrophy of the posterior element of the cervical 
spine (7). Clinical awareness of these deformities can aid clinicians in making early diagnosis of FOP, but quantitative assessment of these deformities has not yet been determined.

In this study, we retrospectively examined radiographs of the hand and cervical spine in FOP patients and demonstrated various abnormal radiographic parameters helpful for early diagnosis of this specific disorder.

\section{Materials and Methods}

\subsection{Demographics}

This study represents a retrospective case-control study consisting of Japanese FOP patients followed up at health care facilities where members of the Research Committee on Japanese Fibrodysplasia Ossificans Progressiva practiced. After approval from the Institutional Review Board of the Nagoya University Hospital, we collected the hand and/or cervical spine radiographs from 18 FOP patients ( 9 males and 9 females) with an average age of 13.9 years (range $0.7-39.3$ years) at the time of this study. The patients were diagnosed clinically and radiographically based on various characteristic findings of FOP including deformities of the great toes, extraskeletal HO, joint contractures, cervical fusions, broad femoral necks, and osteochondroma-like lesions. Molecular testing was performed on fourteen patients. Thirteen showed the common ACVR $1 / A L K 2$ mutation within the glycine/serine-rich regulatory (GS) domain (c.617G > A, p.R206H), and one patient had an atypical mutation within the protein kinase domain (c.774G > T, p.R258S). Molecular studies were not conducted for the remaining 4 patients who showed characteristic skeletal features of FOP. We examined anteroposterior (AP) radiographs of the hands and lateral radiographs of the cervical spine in each individual. The earliest hands and cervical spine films were analyzed using image processing and analysis software Image $J^{\circledR}$.

\subsection{Radiographic assessment of the hand}

According to the measurement method by Poznanski et al. (8), the length of each phalanx and metacarpal bone was measured. In brief, the tangent lines were drawn at both ends of each bone, which were perpendicular to the bone axis, and a bone length was defined as the distance between these two lines (Figure 1). We measured a length of the distal (D1) and proximal (P1) phalanges of the thumb as well as that of the first and second metacarpal bones (MET1 and MET2), and calculated the following bone length ratios, MET2/ MET1, MET2/P1, MET2/D1, MET1/P1, MET1/ $\mathrm{D} 1$, and P1/D1. Radiographs of both hands from one patient were separately analyzed to obtain the average value of the measurements. Reference ranges of these

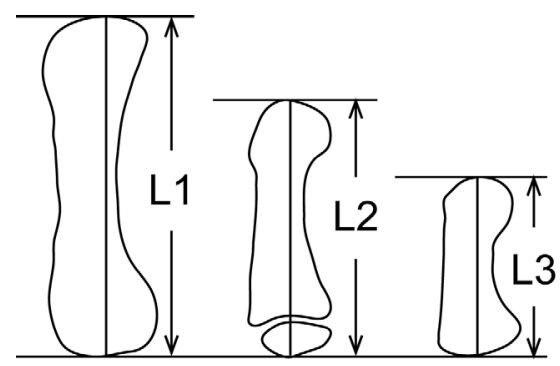

Figure 1. A schematic diagram illustrating the measurement method of bone length in the hand. Bone length was defined as the distance between the tangents drawn to each end of the bone, which were perpendicular to the bone axis. The entire bone length was measured for adults (L1), children (L2), and infants (L3).

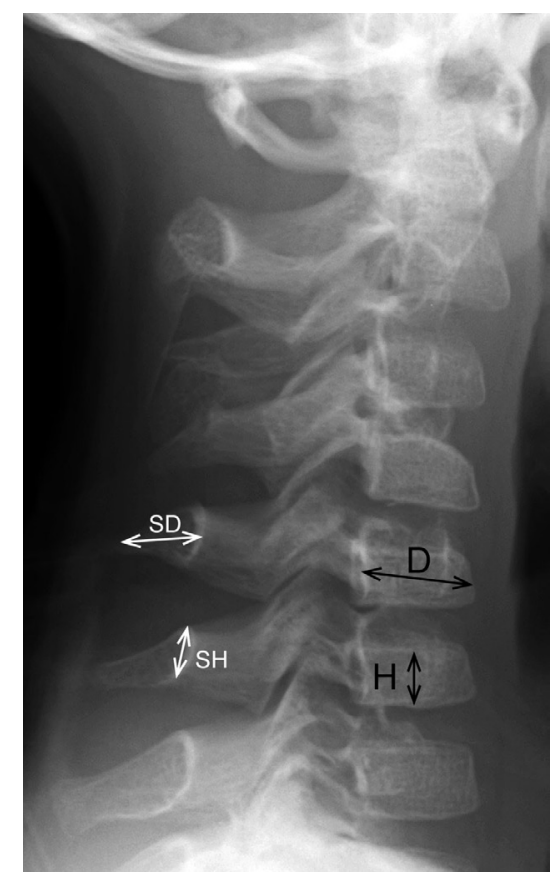

Figure 2. A radiograph depicting the measurements of the bone length in the cervical spine. The height $(\mathrm{H})$ and depth (D) of the C5 vertebral body was measured at the midportion of the body. The height of the $\mathrm{C} 5$ spinous process $(\mathrm{SH})$ was defined as the distance from the cranial to the caudal rim at the juxta-laminar zone. The depth of the spinous process (SD) was measured from the midpoint of the anterior wall to that of the posterior rim.

measurements in different ages and genders were used based on the literature from Poznanski et al. (8). The control data of these measurements in infant $(n=$ 21) were determined by the radiographic database in Nagoya University Hospital.

\subsection{Radiographic assessment of the cervical spine}

According to the measurement method proposed by Remes et al. (9), the height and depth of the C5 vertebral body were measured. Briefly, vertebral body height (H) was measured at the midpoint of the vertebra, perpendicular to the lower end plate. The vertebral body depth (D) was measured at the midpoint of the body from the anterior wall to the posterior wall (Figure 2). The H/D ratios of the C5 vertebra were then calculated 
Table 1. Characteristics and quantitative indices for the study population

\begin{tabular}{|c|c|c|c|c|c|c|c|}
\hline \multirow{2}{*}{ Patient } & \multirow{2}{*}{ Sex } & \multirow{2}{*}{$\begin{array}{c}\text { ALK2 } \\
\text { mutation }\end{array}$} & \multirow{2}{*}{$\begin{array}{c}\text { Age at X-ray (yrs) } \\
\text { Hand/Cervical spine }\end{array}$} & \multicolumn{4}{|c|}{ Deviation of the bone length ratios (SD) } \\
\hline & & & & MET2/D1 & MET2/D1 & $\mathrm{H} / \mathrm{D}$ & $(\mathrm{SH}+\mathrm{SD}) / \mathrm{D}$ \\
\hline 1 & M & $\mathrm{R} 206 \mathrm{H}$ & $0 / 0$ & 1.0 & 1.0 & 0.6 & 0.1 \\
\hline 2 & M & $\mathrm{R} 206 \mathrm{H}$ & $0 / 0$ & 2.4 & 2.4 & 0.6 & 7.1 \\
\hline 3 & M & $\mathrm{R} 206 \mathrm{H}$ & $1 / 3$ & 3.1 & 3.1 & 0.9 & 2.8 \\
\hline 4 & $\mathrm{~F}$ & $\mathrm{R} 206 \mathrm{H}$ & $5 / 6$ & 2.8 & 2.8 & 3.3 & 8.3 \\
\hline 5 & M & $\mathrm{R} 206 \mathrm{H}$ & $8 / 7$ & 6.2 & 6.2 & 2.8 & 3.7 \\
\hline 6 & M & $\mathrm{R} 206 \mathrm{H}$ & $12 / 18$ & 4.1 & 4.1 & 1.9 & 1.5 \\
\hline 7 & $\mathrm{~F}$ & $\mathrm{R} 206 \mathrm{H}$ & $17 / 17$ & 4.0 & 4.0 & 4.1 & NA \\
\hline 8 & $\mathrm{~F}$ & $\mathrm{R} 206 \mathrm{H}$ & 20/NA & 2.2 & 2.2 & NA & NA \\
\hline 9 & M & $\mathrm{R} 206 \mathrm{H}$ & 29/NA & 2.7 & 2.7 & NA & NA \\
\hline 10 & M & $\mathrm{R} 206 \mathrm{H}$ & $34 / \mathrm{NA}$ & 3.5 & 3.5 & NA & NA \\
\hline 11 & $\mathrm{~F}$ & $\mathrm{R} 206 \mathrm{H}$ & $36 / \mathrm{NA}$ & 1.0 & 1.0 & NA & NA \\
\hline 12 & M & $\mathrm{R} 206 \mathrm{H}$ & $39 / 16$ & 1.9 & 1.9 & 3.0 & 1.8 \\
\hline 13 & $\mathrm{~F}$ & $\mathrm{R} 206 \mathrm{H}$ & NA/18 & NA & NA & 0.6 & 2.4 \\
\hline 14 & $\mathrm{~F}$ & $\mathrm{R} 258 \mathrm{~S}$ & $14 / 14$ & 1.7 & 1.7 & 4.9 & NA \\
\hline 15 & M & ND & $\mathrm{NA} / 4$ & NA & NA & 3.2 & 8.8 \\
\hline 16 & $\mathrm{~F}$ & ND & NA/8 & NA & NA & 9.2 & 7.9 \\
\hline 17 & $\mathrm{~F}$ & ND & NA/16 & NA & NA & 4.4 & NA \\
\hline 18 & $\mathrm{~F}$ & ND & $5 / 5$ & 5.3 & 5.3 & 5.3 & 5.4 \\
\hline
\end{tabular}

M denotes male; F, female; ND, not determined; NA, not applicable; SD, standard deviation.

and compared to normal reference values established by Remes et al. in different age and gender groups (9). In addition, we measured the height and depth of the C5 spinous process. The height of the spinous process $(\mathrm{SH})$ was defined as the distance from the cranial to caudal margin at the junction of the spinous process and lamina. The depth of spinous process (SD) was measured from the midportion of the anterior wall to that of the posterior rim demarcating a thick cortex shadow (Figure 2). The sum of SH and SD measurements was used for the evaluation of spinous process size, then the $(\mathrm{SH}+\mathrm{SD}) /$ $\mathrm{D}$ ratio of the $\mathrm{C} 5$ vertebra was calculated. Reference values of the $(\mathrm{SH}+\mathrm{SD}) / \mathrm{D}$ ratio were established from the radiographic database of normal controls in Nagoya University Hospital.

\section{Results}

\subsection{Characteristics of the study cohort}

Patients' characteristics and quantitative indices of the measurements are shown in Table 1. Deviation of the bone length ratios in the hand and cervical spine was calculated based on age-matched reference values.

\subsection{Radiographic characteristics of the hand}

Mean and standard deviation of the MET2/D1 and MET2/MET1 ratio in control infants $(n=21)$ are 2.9 \pm 0.29 and $1.64 \pm 0.08$, respectively. Twenty-six hand radiographs from 14 patients (8 males and 6 females) were available. Regardless of age and gender, all FOP patients showed a MET2/D1 ratio larger than $+1 \mathrm{SD}$ of normal controls (Figure 3A and 3B). In infant patients without an epiphyseal ossification center of the first metacarpal bone, the MET2/MET1 ratio was extremely
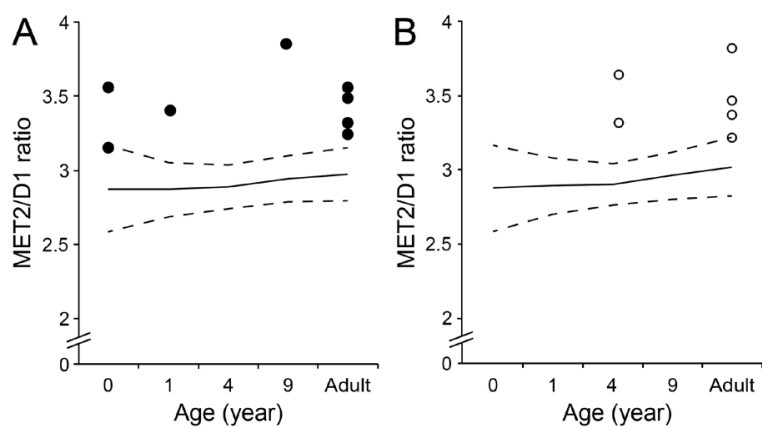

Figure 3. Scatter plots showing the bone length ratio of the second metacarpal bone (MET2) to the distal phalanx of the thumb (D1) in male (A) and female (B) patients with FOP. Solid and dash lines denote the normal value and the standard deviation (SD) of the MET2/D1 ratio, respectively.
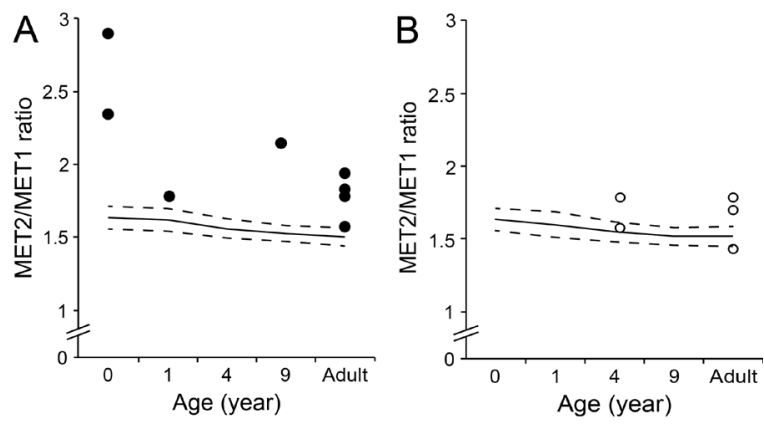

Figure 4. Scatter plots showing the bone length ratio of the second metacarpal bone (MET2) to the first metacarpal bone (MET1) in male (A) and female (B) patients with FOP. Solid and dash lines denote the normal value and the standard deviation (SD) of the MET2/MET1 ratio, respectively.

large ( $>+3 \mathrm{SD}$ of normal controls) (Figure 4A and 4B). The MET2/P1 ratio was higher in infant patients, but it scattered around the mean value with increasing age (data not shown). There were no characteristic features in the values of the MET1/P1, MET1/D1, and P1/D1 ratios in FOP patients, although the MET1/P1 and MET1/D1 
Table 2. Mean and standard deviation of normal controls for the (SH+SD)/D ratio of the $\mathrm{C} 5$ vertebra

\begin{tabular}{lcccccccccccccccccccccc}
\hline Age group & $<1$ & $1-2$ & $2-3$ & $3-4$ & $4-5$ & $5-6$ & $6-7$ & $7-8$ & $8-9$ & $9-10$ & $10-11$ & $11-12$ & $12-13$ & $13-14$ & $14-15$ & $15-16$ & $16-17$ & $17-18$ & $18-19$ & $19-20$ & $20-21$ \\
\hline Mean & 1.05 & 1.10 & 1.0 & 1.20 & 1.21 & 1.43 & 1.37 & 1.47 & 1.33 & 1.47 & 1.50 & 1.53 & 1.51 & 1.57 & 1.69 & 1.86 & 1.76 & 1.73 & 1.71 & 1.78 & 1.86 \\
$\mathrm{SD}$ & 0.13 & 0.15 & 0.15 & 0.13 & 0.11 & 0.18 & 0.18 & 0.17 & 0.19 & 0.18 & 0.16 & 0.18 & 0.20 & 0.19 & 0.12 & 0.16 & 0.22 & 0.22 & 0.23 & 0.24 & 0.25 \\
$\mathrm{~N}$ & 11 & 21 & 17 & 13 & 6 & 13 & 25 & 19 & 20 & 17 & 16 & 17 & 14 & 20 & 16 & 21 & 23 & 31 & 28 & 38 & 20 \\
\hline
\end{tabular}

SD denotes standard deviation; $\mathrm{N}$, number of control subjects; SH, height of the spinous process; SD, depth of the spinous process; $\mathrm{D}$, depth of the vertebral body.
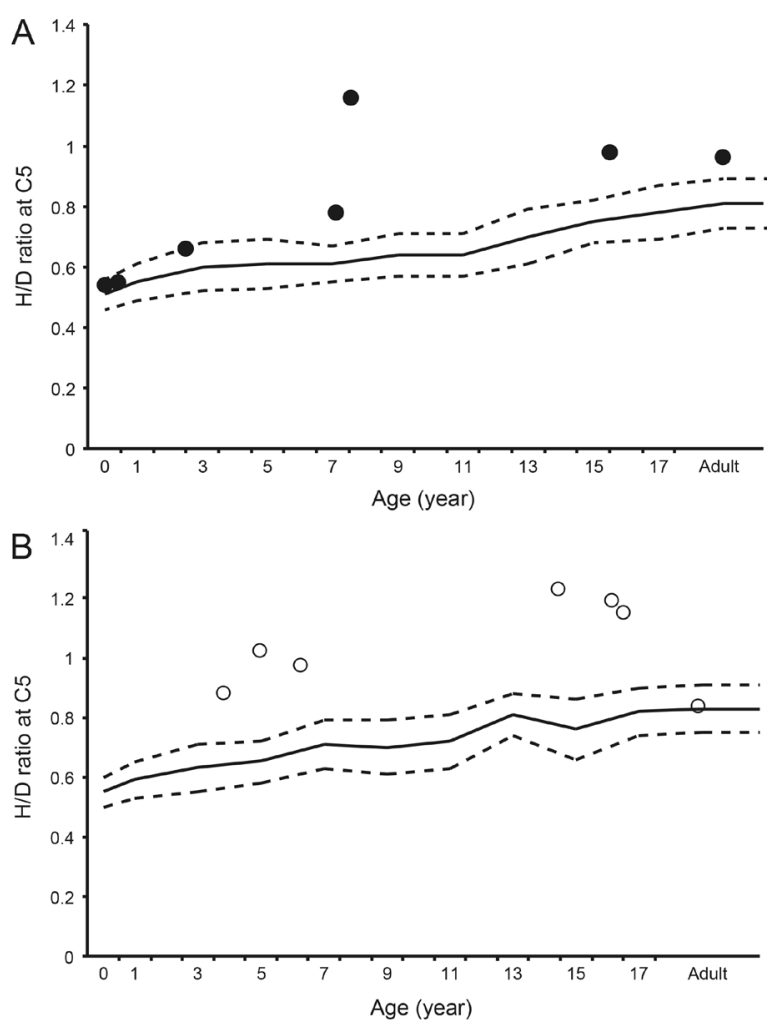

Figure 5. Scatter plots showing the bone length ratio of the C5 vertebral height (H) to depth (D) in male (A) and female (B) patients with FOP. Solid and dashed lines denote the normal value and the standard deviation (SD) of the H/D ratio, respectively.

ratios were relatively small $(<-1 \mathrm{SD}$ of normal controls) in infant FOP patients (data not shown).

\subsection{Radiographic characteristics of the cervical spine}

Reference values of the $(\mathrm{SH}+\mathrm{SD}) / \mathrm{D}$ ratio of the $\mathrm{C} 5$ vertebra are shown in Table 2. There were 14 (7 males and 7 females) cervical spine radiographs available for analysis. Among them, three radiographs were excluded from analysis of the $(\mathrm{SH}+\mathrm{SD}) / \mathrm{D}$ ratio for insufficient resolution. The H/D ratio of the $\mathrm{C} 5$ vertebra exceeded $+2 \mathrm{SD}$ of normal controls in patients over 4 years of age except one female adult patient (Figure 5A and 5B). Similarly, the $(\mathrm{SH}+\mathrm{SD}) / \mathrm{D}$ ratio of the $\mathrm{C} 5$ vertebra was larger than $+2 \mathrm{SD}$ of normal controls in young patients except one male infant (Figure 6).

\section{Discussion}

In the present study, we quantitatively proved the hand

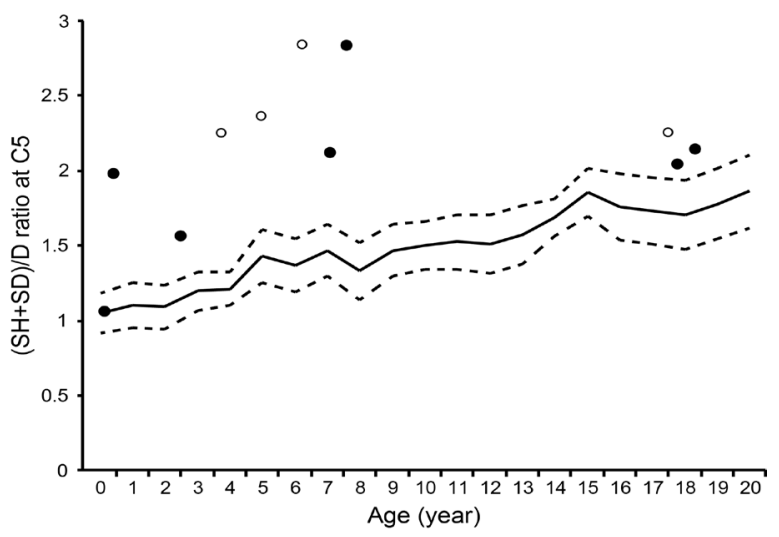

Figure 6. Scatter plots showing the bone length ratio of the $\mathbf{C} 5$ spinous process height (SH) + depth (SD) to the $\mathbf{C 5}$ vertebral depth (D). Solid and open circles indicate male and female, respectively. Solid and dashed lines denote the normal value and the standard deviation (SD) of the $(\mathrm{SH}+\mathrm{SD}) / \mathrm{D}$ ratio, respectively.

and cervical spine abnormalities in FOP including shortened thumbs as well as tall and narrow vertebral bodies and hypertrophic posterior elements of the cervical spine $(7,10)$. Especially in young patients, shortening of the first metacarpal bone and enlargement of the cervical spinous processes were pathognomonic findings useful for early diagnosis of FOP before the appearance of $\mathrm{HO}$.

Previous studies have reported that thumb shortening was seen in $50 \%$ of FOP patients (6). In the present study, all patients had a MET2/D1 ratio larger than +1 SD of normal controls, and $85 \%(11 / 13)$ of the patients showed an increased MET2/MET1 ratio. The thumb shortening, therefore, seems to be more common than previous reports in FOP. Furthermore, an extremely high MET2/MET1 ratio in infant patients suggested that disproportionate shortening of the first metacarpal bone was an important early radiographic finding in FOP (Figure 7).

It is an intriguing feature of FOP that thumb morphogenesis is exclusively disrupted in the development of digit formation (11). The thumb is the last digit in the autopod to form, and it is different from other digits in terms of its relative position, shape, size, and number of phalanges. These unique thumb identities may be attributed to the expression profile of HoxD genes, which are pivotal transcriptional factors regulating limb patterning and growth (12). All four HoxD10 to D13 genes are expressed in the future digit II-V area in the autopod during the hand plate formation, whereas sole expression of the 


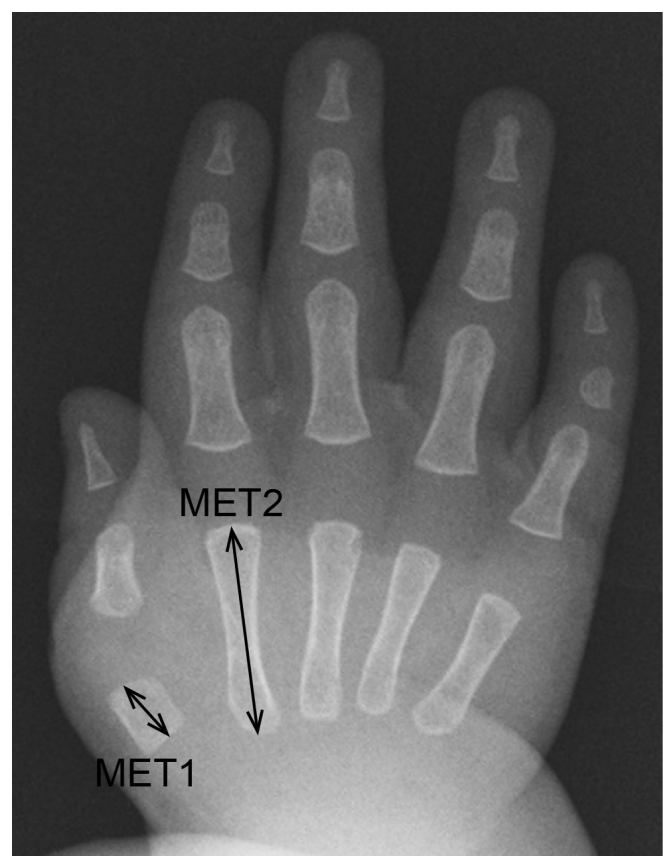

Figure 7. An anteroposterior radiograph of the right hand of Patient 1 at the age of eight months showing marked shortening of the first metacarpal bone. The MET2/ MET1 ratio and the corresponding SD value is 2.9 and 16.3, respectively.

HoxD13 gene in the presumptive digit I area is of great significance (13). Mutations in the homeodomain of the HoxD13 gene cause brachydactyly type D that is characterized by variable shortening of the distal phalanx of the thumb. This mutated HoxD13 proteins responsible for its decreased affinity for the doublestranded DNA target containing a cognitive sequence of the homeodomain (14). Interestingly, previous research has revealed that BMP signaling-dependent Smad1/4 proteins prevented HoxD10 and HoxD13 from binding to DNA targets (15). Constitutivelyactivated BMP signaling in FOP thus is likely to impair HoxD13-mediated transcriptional regulation by direct interactions between BMP-induced Smads and HoxD13. Mesenchymal condensation and chondrocyte proliferation of the presumptive digit I area could be suppressed by down-regulated HoxD13 function, whereas in presumptive digits II to $\mathrm{V}$ areas, it could be preserved by compensating expressions of other HoxD genes (HoxD11 and HoxD12). Dysregulated BMP signal transduction during embryogenesis seems to cause relative shortening of the first metacarpals and distal phalanges of the thumb in FOP.

More than $90 \%$ of adult FOP patients showed fusion of the facet joints, which is a type of orthotopic ossification (6). To our knowledge, however, there are no reports delineating the precise prevalence of tall and narrow vertebral bodies and enlarged posterior elements of the cervical vertebrae. Here we demonstrated that the $\mathrm{H} / \mathrm{D}$ and $(\mathrm{SH}+\mathrm{SD}) / \mathrm{D}$ ratios in the $\mathrm{C} 5$ vertebrae were larger than $+2 \mathrm{SD}$ of normal values in $64 \%$ and $73 \%$ of patients, respectively (Figure 8 ). In addition to

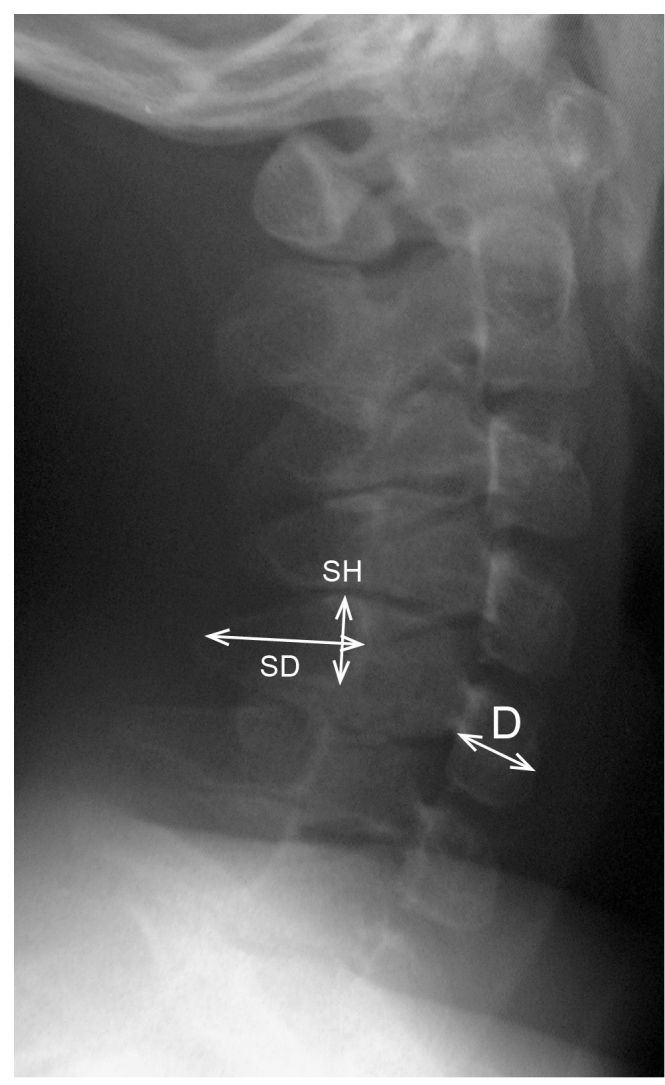

Figure 8. A lateral radiograph of the cervical spine of Patient 16 at the age of eight years showing enlarged spinous process of the $\mathbf{C} 5$ vertebra. The $(\mathrm{SH}+\mathrm{SD}) / \mathrm{D}$ ratio and the corresponding SD value is 2.8 and 7.9 , respectively.

neck stiffness, which seemed to be an important early clinical sign before the appearance of $\mathrm{HO}(6)$, tall and narrow vertebrae and hypertrophic spinous processes of the cervical spine are radiographic characteristics in young FOP patients.

In a previous in vivo study, genetically-engineered overexpression of BMP-2/4 both dorsally and laterally to the neural tube manifested combined phenotypes of hypertrophic spinous processes and large deletion of the lateral and ventral parts of vertebral bodies (16). Thus, mesenchymal condensations at the paraxial mesoderm in FOP, where BMP-2 signaling is aberrantly activating, could be responsible for both enlarged spinous processes and relatively tall vertebral bodies.

The common ACVR $1 / A L K 2$ mutation (c. $617 \mathrm{G}>\mathrm{A}$, p.R206H) shows a homogeneous phenotype including congenital malformation of the great toes and the skeletal features in the thumb and cervical spine (17). In contrast, several atypical mutations in the ALK2/ACVR1 gene, such as L196P, R258S, R375P, G328R, and P197_F198 del insL, have been identified in patients who showed normal-appearing great toes (18). In this study, one patient (Patient 14) with an atypical mutation (c.774G > C, p.R258S) showed normal-appearing great toes. She also lacked the shortened thumb but exhibited exceptionally tall and narrow vertebral bodies. Another patient (Patient 4) who showed neither malformed great 
toes nor shortening of the first metacarpal bone also manifested distinctive features of the cervical spine in spite of the common $A C V R 1 / A L K 2$ mutation. We believe that radiographic characteristics of the cervical spine are potent diagnostic clues for FOP especially in cases without typical deformities of the great toes.

\section{Acknowledgements}

This work was supported partly by Research Committee on Fibrodysplasia Ossificans Progressiva from the Ministry of Health, Labour and Welfare of Japan.

\section{References}

1. Shore EM, Xu M, Feldman GJ, et al. A recurrent mutation in the BMP type I receptor ACVR1 causes inherited and sporadic fibrodysplasia ossificans progressiva. Nat Genet. 2006; 38:525-527.

2. Kitterman JA, Kantanie S, Rocke DM, Kaplan FS. Iatrogenic harm caused by diagnostic errors in fibrodysplasia ossificans progressiva. Pediatrics. 2005; 116:e654-e661.

3. Kitoh H, Achiwa M, Kaneko H, Mishima K, Matsushita M, Kadono I, Horowitz JD, Sallustio BC, Ohno K, Ishiguro $\mathrm{N}$. Perhexiline maleate in the treatment of fibrodysplasia ossificans progressiva: An open-labeled clinical trial. Orphanet J Rare Dis. 2013; 8:163.

4. Pignolo RJ, Shore EM, Kaplan FS. Fibrodysplasia ossificans progressiva: Diagnosis, management, and therapeutic horizons. Pediatr Endocrinol Rev. 2013; 10 (Suppl 2):437-448.

5. Nakashima Y, Haga N, Kitoh H, Kamizono J, Tozawa K, Katagiri T, Susami T, Fukushi J, Iwamoto Y. Deformity of the great toe in fibrodysplasia ossificans progressiva. J Orthop Sci. 2010; 15:804-809.

6. Kaplan FS, Glaser DL, Shore EM, Deirmengian GK, Gupta R, Delai P, Morhart P, Smith R, Le Merrer M, Rogers JG, Connor JM, Kitterman JA. The phenotype of fibrodysplasia ossificans progressiva. Clin Rev Bone Miner Metab. 2005; 3:183-188.

7. Mishima K, Kitoh H, Katagiri T, Kaneko H, Ishiguro N. Early clinical and radiographic characteristics in fibrodysplasia ossificans progressiva: A report of two cases. J Bone Joint Surg Am. 2011; 93:e52.

8. Poznanski AK, Garn SM, Holt JF. The thumb in the congenital malformation syndromes. Radiology. 1971; 100:115-129.

9. Remes VM, Heinanen MT, Kinnunen JS, Marttinen EJ. Reference values for radiological evaluation of cervical vertebral body shape and spinal canal. Pediatr Radiol. 2000; 30:190-195.

10. Kaplan FS, Le Merrer M, Glaser DL, Pignolo RJ, Goldsby RE, Kitterman JA, Groppe J, Shore EM. Fibrodysplasia ossificans progressiva. Best Pract Res Clin Rheumatol. 2008; 22:191-205.

11. Pignolo RJ, Shore EM, Kaplan FS. Fibrodysplasia ossificans progressiva: Clinical and genetic aspects. Orphanet J Rare Dis. 2011; 6:80.

12. Oberg KC. Review of the molecular development of the thumb: Digit primera. Clin Orthop Relat Res. 2014; 472:1101-1105.

13. Deschamps J. Tailored Hox gene transcription and the making of the thumb. Genes Dev. 2008; 22:293-296.

14. Johnson D, Kan SH, Oldridge M, Trembath RC, Roche P, Esnouf RM, Giele H, Wilkie AO. Missense mutations in the homeodomain of HOXD13 are associated with brachydactyly types D and E. Am J Hum Genet. 2003; 72:984-997.

15. Li X, Nie S, Chang C, Qiu T, Cao X. Smads oppose Hox transcriptional activities. Exp Cell Res. 2006; 312:854864.

16. Monsoro-Burq AH, Duprez D, Watanabe Y, Bontoux M, Vincent C, Brickell P, Le Douarin N. The role of bone morphogenetic proteins in vertebral development. Development. 1996; 122:3607-3616.

17. Kaplan FS, Xu M, Glaser DL, Collins F, Connor M, Kitterman J, Sillence D, Zackai E, Ravitsky V, Zasloff M, Ganguly A, Shore EM. Early diagnosis of fibrodysplasia ossificans progressiva. Pediatrics. 2008; 121:e1295-e1300.

18. Kaplan FS, Xu M, Seemann P, et al. Classic and atypical fibrodysplasia ossificans progressiva (FOP) phenotypes are caused by mutations in the bone morphogenetic protein (BMP) type I receptor ACVR1. Hum Mutat. 2009; 30:379-390.

(Received April 13, 2014; Revised April 24, 2014 ; Accepted May 07, 2014) 